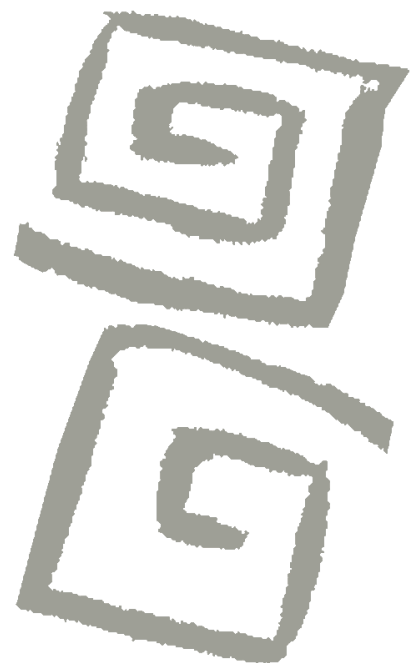

\title{
La dimensión temporal del consumo de drogas: análisis sociológico desde una categoría clave para el estudio de los procesos de salud-enfermedad- atención-cuidado
}

\author{
The temporal dimension of drugs: a sociological \\ analysis based on a category key to the study of \\ health-disease-care processes
}

Victoria Sánchez Antelo ${ }^{1}$

'Socióloga, Doctora en Ciencias Sociales. Becaria posdoctoral, Consejo Nacional de Investigaciones Científicas y Técnicas (CONICET) Investigadora docente, Instituto de Investigaciones Gino Germani, Facultad de Ciencias Sociales, Universidad de Buenos Aires. Investigadora docente, Instituto de Ciencias de la Salud, Universidad Nacional de Tres de Febrero, Argentina.

vsantelo@untref.edu.ar
RESUMEN Se analizan las dimensiones temporales que modulan los sentidos y las prácticas de varones y mujeres policonsumidores de sustancias psicoactivas de 18 a 35 años residentes en el Área Metropolitana de Buenos Aires. Desde un enfoque cualitativo, se realizaron 29 entrevistas en profundidad individuales, analizadas mediante un proceso de comparación constante de análisis entre las categorías generadas a partir de los datos y conceptos teóricos. Del análisis emergen prácticas y sentidos que regulan las diversas temporalidades que atraviesan el consumo de drogas, como los sentimientos vinculados a los ritmos corporales, las frecuencias entre cada consumo, las fases del ciclo de vida en las que se va a contratiempo, o las temporalidades inespecíficas que vuelven a un "momento" adecuado para el consumo. Prácticas que requieren una especial atención sobre el tiempo para denotar la flexibilidad que les permita consumir sin ser consumistas, drogarse sin ser adicto.

PALABRAS CLAVES Consumidores de Drogas; Tiempo; Sociología; Argentina.

ABSTRACT The temporal dimensions that shape the senses and practices of men and women who are poly-consumers of psychoactive substances, 18-35 years of age, and living in the metropolitan area of Buenos Aires were analyzed. Using a qualitative approach, 29 individual in-depth interviews were carried out and then analyzed through a constant comparative analysis process between the categories generated from the data obtained and the theoretical concepts. From the analysis, practices and meanings emerge that regulate the diverse temporalities that underlie drug consumption: feelings related to body rhythms, periods between consumptions, the timing of phases of the life cycle, or unspecific temporalities that become an adequate "moment" for consumption. These practices require that particular attention be paid to time, as this enables the flexibility to consume without being a consumer, to use drugs without being addicted to them.

KEY WORDS Drug Users; Time; Sociology; Argentina. 


\section{INTRODUCCIÓN}

La importancia sobre el modo en que los grupos humanos viven, sienten y conciben el tiempo, no es nueva. Tanto las ciencias sociales como la filosofía han reflexionado extensamente sobre este concepto y un conjunto amplio de términos de los cuales es su eje de gravitación ${ }^{(1),(2),(3)}$. En el campo de la salud, numerosas líneas de análisis son ejemplo del carácter intrínseco del tiempo en el proceso salud-enfermedad-atención-cuidado (PSEAC):

a) La noción misma de proceso marca este vínculo, en contraste con las visiones estáticas y a-temporales. Reconocer este corrimiento de lo "estático" a lo "dinámico" ha dado relevancia a los estudios tendientes a comprender la historicidad del PSEAC y de los colectivos que lo protagonizan.

b) Al analizar los aspectos institucionales y organizacionales en torno a interrogantes sobre la "calidad", la "satisfacción" y la "planificación" se puede reconocer una multiplicidad de temporalidades: los tiempos de espera, el tiempo destinado a la consulta, los modalidades horarias para "gestionar" los recursos humanos en salud, así como la estructuración de los reclamos de los trabajadores de la salud en torno a lo insalubre de los regímenes temporales que impone la creciente mercantilización de los sistemas sanitarios $^{(4),(5)}$.

c) Al cuantificar el tiempo destinado a otros, para dar cuenta de la dimensión del "cuidado", se ilumina un rasgo nefasto de nuestra sociedad, pero no por ello obvio, que es la persistente inequidad de género ${ }^{(6)}$.

d) Al reflexionar sobre la especificidad de las enfermedades crónicas, la percepción de paréntesis temporal que supone una enfermedad y su tratamiento en una trayectoria de vida más amplia, o el hito temporal que determinados eventos de la vida, como el nacimiento de un/a hijo/a, en los que participan los equipos de salud, pueden estructurar la percepción de la propia biografía ${ }^{(7)}$.

e) La multiplicidad de temporalidades contenidas en las definiciones epidemiológicas que se utilizan cotidianamente, fuertemente ancladas en la concepción del tiempo lineal, objetivo y externo a las personas y los colectivos sociales en estudio.

f) La relevancia de los ciclos de vida, las edades o generaciones asociadas al PSEAC. g) El uso de escalas de "percepción del tiempo" que han sido conceptualizadas y estandarizadas para relevar daños o "anomalías" en el proceso cognitivo que diferencia pasado/presente/futuro ${ }^{(8)}$.

No se trata de un listado exhaustivo, pero permite dar cuenta de las diversas formas en que el tiempo y las temporalidades atraviesan las investigaciones y las prácticas del campo de la salud en general, y de los propios análisis de la sociología médica o de la salud, en especial.

El propósito de este trabajo es poner el acento en la necesidad de reflexionar acerca de las diversas concepciones del tiempo que están puestas en juego en el PSEAC. Este propósito toma sentido si se reconocen las reconfiguraciones temporales de lo que se ha dado en Ilamar la modernidad tardía o modernización reflexiva, que han puesto en tensión las diversas actividades cotidianas y cuyo atributo más destacado es el proceso de aceleración social ${ }^{(9),(10)}$.

Existe un conjunto de ordenamientos, clasificaciones y jerarquías temporales que son socialmente compartidos. No compartirlos, o no cumplir la norma sociotemporal vigente, convierte el comportamiento del infractor en sospechoso y, por tanto, desviado o problemático ${ }^{(11),(12)}$. Con la atención en el uso de drogas, en este trabajo se observa que para definir los consumos problemáticos se utiliza una batería de criterios que se sustentan en concepciones temporales jerárquicas (el empleo por sobre otros tiempos), divididas en compartimentos estancos (de lunes a viernes vs. sábado y domingo) y secuenciales (que no reconocen la simultaneidad). También, entre las personas entrevistadas, estos valores son definidos como un "indicador de integración social". Comparten la concepción por la cual no cumplir estas normas temporales, ir a destiempo, es un problema. 
Si el tiempo es un clasificador de las prácticas en tanto normales/adecuadas y anormales/inadecuadas, en el plano de la experiencia cotidiana de jóvenes de sectores medios del Área Metropolitana de Buenos Aires $(\mathrm{AMBA})^{[\mathrm{a}]}$, la reconfiguración y aceleración temporal actual ¿qué tensiones expresan en sus prácticas cotidianas?, ¿qué estrategias despliegan para "controlar" estas temporalidades en el contexto del consumo de drogas? En las formas de clasificar lo problemático y lo no problemático en torno al consumo de drogas iredefinen los criterios temporales o a partir de una redefinición temporal reubican lo problemático en otro espectro de lo social?

El objetivo de este trabajo es, por lo tanto, analizar las dimensiones temporales que modulan los sentidos y las prácticas de uso de sustancias psicoactivas en varones y mujeres policonsumidores de 18 a 35 años, de sectores socioeconómicos medios y residentes en el AMBA durante el período 2011-2013.

A continuación se introducen las nociones teóricas que permitirán, luego de la descripción metodológica, analizar los datos empíricos obtenidos.

\section{Sociología del tiempo y de las temporalidades}

Desde la perspectiva sociológica del tiempo, Émile Durkheim ${ }^{(13)}$ sostiene que la experiencia individual, que se sostiene en la distinción entre un momento y otro, es una institución social destinada a garantizar el ritmo y la regularidad colectiva. Los ritos, el calendario y las festividades, sean laicas o religiosas, son indicios de esa institucionalización social del tiempo. Pitirim Sorokin y Robert Merton ${ }^{(14)}$ cuestionan las definiciones de tiempo ancladas en la astronomía, que lo conceptualizan como homogéneo, cuantitativo y lineal. Por el contrario, sostienen que el tiempo social es "discontinuo", marcado por "lagunas", hitos cargados de sentidos -festividades o eventos vitales- y períodos temporales significados como "vacíos". Estas críticas pretendían resaltar la relación entre la significación individual del tiempo y de las diversas temporalidades, con las definiciones y actividades sociales ${ }^{(2)}$.

Desde el interaccionismo simbólico, Lewis y Weigert ${ }^{(15)}$ enfatizaron tres aspectos del tiempo social. El primero, la estratificación o diferenciación entre temporalidades, que supone comprender los distintos niveles temporales: individual (nivel subjetivo), institucional (nivel social) y ciclos temporales (nivel cultural). El segundo, la integración (embeddedness) de las interacciones cotidianas de cada persona en una organización temporal más amplia; esto posibilita el sentimiento de continuidad de un ser que protagoniza diversas y fragmentarias acciones diarias; la experiencia del paso del tiempo más veloz está relacionada con una mayor integración social de las personas. Por último, el tercer aspecto, la sincronización que establece la relación entre las categorías anteriores: sincronizar de un modo comprensible la experiencia de la temporalidad individual con el tiempo colectivo que, a su vez, implica interacciones en diversos estratos temporales.

Se puede hacer notar que, hasta aquí, no se ha señalado una dimensión conflictiva entre los niveles temporales y hacerlo supone reconocer jerarquías entre los diversos "estratos". Desde las perspectivas feministas, se ha denunciado la subordinación y desvalorización de los tiempos de la reproducción social, protagonizados en mayor medida por mujeres, frente a los tiempos productivos, marcadamente masculinos. Incluso, la mayor presencia femenina en el mundo del empleo ha dado lugar a la doppia presenza ${ }^{[b]}$ con la consecuente sobrecarga laboral ${ }^{(16),(17)}$. Las jerarquizaciones entre temporalidades han sido fruto de un proceso histórico mediante el cual se ha naturalizado la idea moderna de tiempo.

La posibilidad de su medición mediante un artefacto "mecánico/categórico" como el reloj ${ }^{(18),(19)}$ facilitó la ficción de un tiempo externo, neutro, único y, por tanto, objetivo. Este proceso supuso, por un lado, la "desnaturalización" -en el sentido de perder su vínculo con los ciclos naturales-y la "racionalización" 
-haciéndolo cuantificable y asociándolo al dinero como unidad de medida ${ }^{(20),(21)} ; y$, por otro, la "normalización" del tiempo productivo/económico como eje ordenador de lo social, lo cual ha sido crucial en el desarrollo del capitalismo industrial para estandarizarlo como "tiempo de trabajo"(22).

De acuerdo al análisis de Michel Foucault sobre los dispositivos disciplinarios, debe reconocerse la centralidad de la dimensión temporal en esta "técnica de ejercicio del poder $^{\prime \prime(23)}$. Según este autor, la disciplina es un modelo de acción sobre los cuerpos, en la que el uso del tiempo deviene en un programa que penetra en el cuerpo estableciendo ritmos, obligando a determinadas ocupaciones y regulando los ciclos de repetición ${ }^{(24)}$. La disciplina establece un régimen temporal de constitución de sujetos "... por el cual la fuerza del cuerpo está con el menor gasto, reducida como fuerza 'política', y maximizada como fuerza útil'(24). Es por lo que algunos autores, que retoman la gubernamentalidad foucoultiana ${ }^{(25)}$, señalan que el tiempo es la sustancia ética de la modernidad reflexiva ${ }^{(26),(27),(28)}$.

El planteo del tiempo como "sustancia ética" exige introducir conceptualizaciones acerca de la percepción subjetiva del tiempo que, lejos de concebirlo como una entidad "natural" y exterior, reconocerá una multiplicidad de sentidos colectivos que son incorporados por el individuo en diversas prácticas. Las mismas acciones adquieren sentidos diversos o contrarios según el tiempo en el que son desarrolladas. Estos sentidos provistos por el ritmo social son transmitidos en tiempos-espacios específicos -ritos, celebraciones, festividades- en los que se produce una condensación de los sentidos y de las emociones colectivas, y se despliega la habilidad aprendida que se transmite a otros. Es un proceso colectivo de aprendizaje, una oportunidad para internalizar las distinciones fundamentales del orden social ${ }^{(12),(29)}$. Desde la concepción de habitus de Bourdieu, no como una repetición mecánica -que implicaría hablar de hábito- sino como disposiciones generadoras, creadoras, el sentido del tiempo, sus ritmos y su tempo se inscriben en la hexis corporal, en esa existencia humana que es el "habitus como lo social hecho cuerpo $^{\prime \prime(30)}$. Los sentidos temporales, transmitidos en la socialización, no se vinculan con una dimensión racional-consciente sobre la que se reflexiona en cada acción, sino con una expertise práctica que se preserva en los prerreflexivos. Son sentidos encarnados, aprendidos por un sutil trabajo pedagógico, mediante una práctica política que prescribe y transmite una práctica, la cual "juega estratégicamente con el tiempo y en particular con el tempo"(11). No se trata de "conocer", sino de haber encarnado una información que va más allá de lo discursivo, que reside en el espectro de la práctica prerreflexiva -como el ritmo de los gestos o de las palabras-, que supone un sentir y un saber llevar corporalmente el tempo social compartido y no solo conocerlo teóricamente ${ }^{(11)}$.

\section{ASPECTOS METODOLÓGICOS}

El trabajo se basa en un estudio exploratorio más amplio ${ }^{[c]}$, del cual se presenta una de las dimensiones vinculada al objetivo enunciado en la introducción. Utilizando un enfoque cualitativo, el trabajo de campo se compuso de 29 entrevistas individuales en profundidad, en las cuales se buscó rescatar -no sin limitaciones- el punto de vista de los protagonistas $^{(31)}$. La guía de preguntas estuvo orientada por interrogantes que pretendían analizar las actividades cotidianas, usos del tiempo y criterios para la delimitación de temporalidades (ordenamientos, clasificaciones y jerarquizaciones), e identificar en esos usos del tiempo el lugar asignado al consumo de drogas. En los relatos se buscó identificar esas temporalidades relacionadas con el consumo de drogas y describir las trayectorias de consumo, para lo cual se indagó en los sentidos que estas tenían para las personas entrevistadas.

Respecto a la selección de casos, el rango de edades se fijó a partir de las prevalencias de consumo de la población del $\mathrm{AMBA}^{[\mathrm{d}]}$, y se privilegió a mayores de 18 años para evitar la incorporación de menores que requieran 
la autorización de adultos responsables. El tamaño final de la muestra se determinó por la saturación en los conceptos centrales para el análisis ${ }^{(32)}$, y quedó conformada por 15 mujeres y 14 varones de sectores medios ${ }^{[\mathrm{e}]}$, policonsumidores de sustancias psicoactivas ${ }^{[f]}$.

Las entrevistas se realizaron entre marzo de 2011 y abril de 2013, y se registraron en audio, previo consentimiento de los participantes, para su posterior trascripción textual. Al momento del contacto, se informó a los entrevistados sobre los objetivos del estudio, se garantizó la confidencialidad y el anonimato, así como el uso exclusivo de los datos para la investigación. Los criterios éticos utilizados siguieron los lineamientos fijados por la Resolución 2857 del Consejo Nacional de Investigaciones Científicas y Técnicas (CONICET) ${ }^{(34)}$.

La técnica de análisis requirió realizar, de forma simultánea a la recolección de datos, la comparación y revisión constante de los datos obtenidos con los conceptos teóricos elaborados, así como la codificación abierta, mediante la cual se identificaron conceptos, sus propiedades y dimensiones, para luego trabajar por ejes temáticos (codificación axial) ${ }^{(35)}$. En esa fase fue posible establecer relaciones entre los códigos abiertos (tipos de ordenamientos temporales que rigen el cotidiano de estos entrevistados, flexibles o fijos $^{[\mathrm{g}]}$ ) y adjetivos, metáforas y explicaciones vinculadas a clasificaciones, ordenamientos y jerarquizaciones temporales utilizadas en la descripción y reflexión acerca de sus prácticas de uso de drogas. Se analizó el corpus buscando definir una trama que posibilitara la comprensión de los hechos y significados socialmente construidos, enunciados por actores individuales ${ }^{(31),(35)}$.

\section{ANÁLISIS DE LOS HALLAZGOS: TEMPORALIDADES Y DROGAS}

\section{Temporalidades largas: la "evolución" como consumidor}

Cuando se hace referencia a la etiología de las adicciones o "usos problemáticos" de drogas parece dominar una concepción que la ubica en una "esencia subjetiva" adicta. Si bien la conducta puede modificarse, la estricta abstinencia se corresponde con la imposibilidad de transformar esa condición $\mathrm{n}^{[\mathrm{h}]}$. En contraste con esta realidad inmutable, los entrevistados describen pautas de consumo como si se tratara de una trayectoria que, con el paso del tiempo, va mutando junto con las transformaciones que la persona va atravesando en sus ciclos de vida. La "búsqueda" de nuevas sustancias, la migración de una a otra, demuestra que "uno va evolucionando, empieza tomando cerveza, después pasa al vino, después al whisky" (E14: Varón, 33 años). Esos "cambios" son un indicio de no haberse "estancado", de no estar "fijado" o ser rígido, aspecto indicado como una característica negativa para este colectivo. Es una búsqueda que denota la capacidad de surfear de forma "estable" por las superficies ${ }^{(36)}$, ya que "caer en una sustancia" es identificado como algo problemático. Según sus términos, "hay diferentes momentos en la vida para tomar diferentes drogas" (E12: Mujer, 26 años). El consumo de drogas se describe como una práctica que cumple una funcionalidad distinta según las etapas del ciclo de vida. El inicio del consumo, según expresan los entrevistados, se produce en la adolescencia como un "riesgo necesario", funcionalidad que ha sido analizada como "rito de paso", y que puede leerse como el despliegue de prácticas que permiten "entrar en el juego"(11),(30) y ser un joven integrado: "en la adolescencia, si no tomas, sos un extraterrestre..." (E9: Mujer, 23 años). Iniciarse en el consumo de drogas fuera de tiempo, "empezar de grande" (E17: Mujer, 23 años), es considerada una práctica problemática. Así, se torna una práctica inevitable y necesaria para la adolescencia:

...en los primeros años te ayuda a ubicarte en un lugar y a "pertenecer $a^{\prime \prime}$... Ahora tomás porque te gusta... ahora sabes con quién, cómo y cuándo. (E4: Mujer, 24 años) 
En la socialización se encarnan esquemas de percepción que permiten llevar a cabo conocimientos prácticos dotados de sentido, que orientan las "opciones" que, sin ser deliberadas, poseen sistematicidad. Es un saber hacer y saber ser que posibilitan el ajuste anticipado a las exigencias de un espacio social, y que suponen, por ejemplo, haber encarnado el "sentido de la ubicación"(11). El consumo de sustancias, más allá de la etapa adolescente, asume otros significados que demuestran el desarrollo de la expertise, saber consumir, pero también "saber cambiar":

\section{Aprendes a cambiar "el por qué" más que lo que se consume ¿no? Cambiar uno y no la sustancia, sino la perspectiva que uno tenga al respecto de lo que uno consume. (E26: Mujer, 26 años)}

Más que denotar una esencia desviada, cristalizada en la idea de un sujeto adicto, se pone en juego una flexibilidad para adecuarse a los cambios del contexto según la etapa de vida. Se trata de un proceso reflexivo sobre el uso del tiempo, en el que se debe aprender a consumir de otro modo o dejar de hacerlo en determinados ámbitos. De allí la idea de nightology ${ }^{[i]}$, que implica saber transitar "la noche", compartimentar las diversas temporalidades coexistentes según la etapa del ciclo de vida, así como reconocer el momento en el que hay que introducir cambios en las prácticas de consumo. Esta expertise es una clave para la delimitación entre consumos problemáticos y no problemáticos desde la perspectiva de estos entrevistados.

\section{Temporalidades concretas: el arte del momento}

Históricamente, el tiempo de ocio es el privilegiado para el consumo de sustancias psicoactivas, algo que lo ha convertido en un campo de tensiones ${ }^{(22),(37)}$. El proceso modernizador supuso el control creciente sobre los tiempos sociales, de modo que las prácticas de ocio no "enturbien" los desempeños productivos de los trabajadores ${ }^{(22)}$. En la sociedad de consumo, el ocio es revalorizado en todas sus dimensiones temporales, y es orientado a la adquisición de bienes y servicios. Así, toman relevancia los instantes, la repetición, la simultaneidad y la aceleración como lógicas prácticas de uso de los tiempos.

Para los entrevistados, los "momentos adecuados" para el consumo de drogas son temporalidades inespecíficas -“cuando pinta" o porque "pintó hacerlo"-, que no deben ser premeditadas o planificadas. Se valora la emergencia de lo inesperado, la "oportunidad" no buscada que habilita a un proceso de decisiones (consumir o no y qué), que se realiza sin un esquema prefijado pero no por ello compulsivo.

\section{P: ¿Hay alguna sustancia que hayas con- sumido en otro momento y después de- jaste de consumir? \\ R: No, probé una vez pepa, pero no es que la deje de consumir... simplemente no volvió a pintar. Porque para mí pasa más por un momento en el cual pinta. La pepa tiene un efecto que está bueno que se dé en momentos en los cuales real- mente estés seguro de que la vas a pasar bien... no se volvió a dar, nada más. \\ (E13: Mujer, 25 años)}

En la descripción que realizan los entrevistados, la referencia a temporalidades inespecíficas -“cuando pinta”- pueden interpretarse como en oposición a nociones binarias que reducen las conductas al par inconsciente/compulsivo frente a la racionalidad del no-consumo si el "momento" no es el adecuado. Cabe destacar la oposición que hacen los entrevistados entre "elección" (vinculado a una inmediatez) y "planificación" (vinculado a premeditación). Estrictamente, la planificación supone la definición de un objetivo, para el cual es necesario el reconocimiento de una serie de pasos y/o factores que, conjugados, permitirían alcanzarlo.
P: ¿Y elegís el momento, el lugar y con quién consumir o es algo más que se presenta la oportunidad y se da? R: No, trato de elegirlo. 
P: De elegirlo... ¿hay una planificación? R: No sé si una planificación, pero... es una elección. (E18: Varón, 30 años)

Por el contrario, la elección de un momento de consumo, sin tratarse de algo plenamente espontáneo, conserva la latencia de la acción por preferencias. Cuando se reconoce una serie de elementos contextuales, a partir de los esquemas de percepción y apreciación aprendidos mediante experiencias acumuladas, es posible desplegar actos de conocimiento práctico ${ }^{(38)}$. Se trata de los saberes aprendidos y preferencias latentes que entran en juego para desencadenar la acción del consumo.

P: ¿Elegís el momento, el lugar y con quién consumir, o se presenta la oportunidad y aprovechás?

R: No, yo creo que... no, yo creo que lo elijo. Bah... si se me presenta la oportunidad también, un poco de cada cosa. Es que a veces se presenta la oportunidad, pero si no están todas las circunstancias dadas y uno sabe que va hacer cagadas, o sería al pedo, no lo hace. Más que nada se planifica. Una mínima planificación... tampoco nada demasiado organizado pero... y también si se da, si se da hay que analizar las circunstancias, pero también se puede dar espontáneamente... (E24: Varón, 23 años)

Frente al ejercicio reflexivo que implica la situación de entrevista, la descripción de las prácticas muestra un equilibrio inestable entre el deseo de la espontaneidad y el reconocimiento de una evaluación previa del escenario potencial de consumo. Ese vaivén entre lo planificado/organizado/premeditado y lo espontáneo/elegido/preferido encierra al conjunto de sentidos prácticos -habitus flexible ${ }^{[j]}$ - que posibilita un "consumo socialmente integrado". Este habitus expresa un saber hacer y, por tanto, un saber ser flexible de acuerdo a parámetros inespecíficos que dan sentido a un "momento" adecuado para el consumo o no. En este sentido se interpretan como prácticas que no son sometidas a un examen consciente, en cada momento, sino incorporadas -hechas cuerpo ${ }^{(11),(38)}-y$ que se despliegan para poner en marcha un consumo que reviste algún tipo de rédito o placer.

Ahora bien, la falta de parámetros temporales, fruto de la flexibilidad temporal imperante, en algunos casos implica no saber cuándo se tendrá "tiempo libre". En el paradigma de la flexibilidad temporal total ${ }^{(10)}$, el ocio intersticial está condicionado por la necesidad de estar disponible, borrando los límites temporales entre lo laboral (público) y lo recreativo (privado), algo que dificulta reconocer los momentos adecuados para el consumo.

Igual... una vez fui a trabajar muy loca... una vez estuve de franco y me llamaron... así de un momento a otro para que cubra un turno, y... [risas] salieron programas al aire... porque yo estaba totalmente del orto, zarpada... y salieron muy mal... [risas]. (E12: Mujer, 26 años)

El tiempo funciona como clasificador social $^{(11),(12)}$, regulador de la moralidad hegemónica ${ }^{(39)}$, excluyendo a quienes van a contratiempo de actividades hegemónicas. La jerarquización temporal de la modernidad ha establecido que lo propio del "tiempo público" son las prácticas que demuestran la habilidad de la responsabilidad -hacia el empleo y estudio- que implica la destreza del "dominio de las pasiones"(40),(41). Mientras que lo propio del tiempo privado es todo lo reservado a las prácticas sometidas a la "observancia de Dios" ${ }^{\prime k]}$ y a la propia conciencia, como sostiene el Artículo 19 de la Constitución Nacional. La imposición de la flexibilidad pone en evidencia que se vive a destiempo y obliga a una reflexión sobre los sentidos asignados a los usos del tiempo y a las prácticas desarrolladas en estos.

Al momento de "elegir" el consumo en una instancia temporal privada, para los entrevistados, la reflexión requiere de una evaluación sobre el tiempo necesario para que pase el efecto y poder "volver a lo público", de lo contrario, es no "saber" consumir. Sin 
embargo, en contextos de hiperflexibilidad temporal, el mandato de disponibilidad permanente pone en tensión al par público/ privado $^{(19)}$. Las prácticas de ocio privadas son reconfiguradas por las exigencias de un trabajo en el que impera la flexibilidad horaria, llevando situaciones y "estados privados" vinculados al consumo de drogas a los ámbitos públicos, algo que no es del orden de lo deseado por los entrevistados.

Sin barreras temporales claras entre ocio privado y responsabilidad pública: ¿qué margen de previsión se puede tener para que prácticas privadas no afecten una integración social normalizada? Destacar la centralidad de la temporalidad permite visibilizar cómo estos desajustes marcan la arbitrariedad de lo arbitrario.

\section{Los ciclos de consumo: frecuencia y consumo que "va por épocas"}

Los "momentos" concretos de consumo de drogas adquieren una periodicidad o ciclos, que introducen una nueva regulación temporal. Según lo enunciado por los entrevistados, estos se pueden estructurar por cuatro factores: las dinámicas del mercado de sustancias; los períodos cambiantes en las actividades cotidianas; la oferta de eventos festivos; y el control, por parte del consumidor, de la frecuencia del uso de drogas.

En primer lugar, el ingreso constante de nuevas sustancias al mercado indica una regulación por "modas". Esto establece etapas en las que un consumo se convierte en una excentricidad de pocos, para luego extenderse. Por otra parte, la disponibilidad de las sustancias en el mercado está signada, según los relatos de las entrevistas, por la escasez de sustancias de "buena calidad". Esto obliga a disminuir los consumos o bien cambiar a otras sustancias. La evaluación de la calidad hace que productos accesibles en el mercado, pero que no cumplen con el estándar requerido por el consumidor, sean descartados como elección, situación que se describe como "de escasez".
Lo más fácil es el porro... que lo podés encontrar en cualquier parte, pero conseguir bueno también es difícil... Lo que pasa es que... es difícil, cada vez que decís "Uy, me gustaría consumir tal cosa" porque querés conseguir algo bueno que no te haga mierda. (E12: Mujer, 26 años)

El interrogante sobre la calidad ha generado, específicamente entre los consumidores de marihuana entrevistados, prácticas de autocultivo. Si bien este se orienta al consumo individual, y a proveer a algunos "amigos", también se asocia a un discurso político sobre el rol del consumidor en la "cadena productiva" de las sustancias. Se describen prácticas que denotan un proceso de reflexión en torno a qué se consume y qué papel cumple el consumidor en el conjunto de acciones que llevan al consumo. Esta "concientización" acerca de la calidad del consumo va en sintonía con tendencias internacionales, en las que grupos de consumidores promueven mercados regulados y políticas de reducción de riesgos y daños, y "conocer" la composición de la sustancia es un elemento relevante para evaluar si se consume o no ${ }^{(42),(43),(44)}$.

En segundo lugar, aquellos que asisten a programas de estudio, a lo largo de un año calendario, verán alteradas sus rutinas por el inicio y el fin de las actividades formativas que liberan o restringen el tiempo para el ocio. Esto produce ciclos de mayor o menor consumo, que se asemejan a la idea de estaciones del año, sin estar estrictamente asociadas a lo climático.

El tercer factor, que introduce una lógica cíclica en las prácticas de ocio, es la participación en eventos masivos. Es una temporalidad impuesta por el mercado del ocio, que promueve ciertas reglas de juego a las que todos deben adecuarse ${ }^{(45)}$. El modelo típico de oferta privada de espectáculos, para los cuales se ha naturalizado la combinación entre música, baile y consumo de drogas, son los grandes festivales de música ${ }^{(43),(46),(47),(48),(49)}$. Sobre la participación en estos eventos, cabe señalar que en estudios sobre jóvenes y consumos suele asociarse monolíticamente una 
estética -musical o de indumentaria- a una serie de prácticas, entre ellas el consumo de determinadas drogas. Así, ser partícipe de festivales de música electrónica se ha convertido en "ser consumidor de éxtasis", como los hippies fueron asociados a la marihuana y los "rockeros" a la cocaína. Por el contrario, según afirman los entrevistados, tanto el consumo de espectáculos como de drogas "van por épocas" (E24: Varón, 23 años) y son fruto del tránsito por múltiples espacios, sin que ello se traduzca en una adhesión identitaria. En este sentido, pueden definirse como policonsumidores, no solo de drogas, sino también de espacios de ocio y estéticas musicales diversas, según la época.

El cuarto factor, que define ciclos de consumo de drogas, se deriva de una reflexión activa de los propios consumidores sobre la frecuencia con la que se produce un consumo respecto de otro. En la reflexión sobre sus prácticas toma relevancia el control de los tiempos con la intención de establecer una gradación en la intensidad de las actividades de ocio, lo que se traduciría en una regulación de los consumos:

...las salidas nocturnas van variando. En el mes, si hay dos o tres, alguna es digamos descontrolada, otra no tanto y otra es tranquila. (E24: Varón, 23 años)

La delimitación de ciclos, según las experiencias sucesivas, permitiría evaluar las transformaciones sobre sí que pueden devenir, o no, en "adicción", según los términos de los entrevistados. Se trata de regular para mantener el consumo en una trayectoria temporal más larga, sin que esto implique una habituación del organismo.

P: ¿Qué es lo que puede motivar dejar de consumir? ¿Te lo planteaste alguna vez? R: Sí, cuando los malos viajes se empiezan a repetir, yo hago una pausa. Cuando veo que me está interfiriendo con... con la vida social, hago una pausa. Yo cuando fumo soy muy consciente de que estoy fumada. O sea, no es que me creo que ando lo más bien, me doy cuenta que puedo llegar a decir boludeces. De hecho, cuando fumo cada tanto, chequeo. Le pregunto a alguien "Che iestoy diciendo muchas boludeces?" Como que bueno... en el momento que no me plantee esas cosas voy a tener que replantearme a ver si... si dejo o si paro un poco. (E23:

Mujer, 25 años)

Se describen prácticas de consumo sobre las que se despliega un proceso reflexivo que parece querer posicionar al sujeto que enuncia fuera del espectro definido como "acción compulsiva". Esto requiere, por una parte, de información cada vez más precisa sobre los efectos de las sustancias, lo que permitiría prever los modos de uso y los tiempos que deben tenerse en cuenta entre un consumo y otro, así como a qué síntomas corporales prestar atención. Al describir estas acciones de "automonitoreo", independientemente de su efectividad, se pueden identificar regulaciones temporales destinadas a reducir los riesgos de convertirse en adictos. La persistencia en el consumo, en el sentido de "engancharse a una droga", con la posible habituación fisiológica, así como el despliegue de prácticas repetitivas sin controlar la frecuencia e intensidad del consumo, es definido como problemático.

\section{Temporalidades micro: regular el ritmo y sincronizar el consumo}

Por último, se pueden destacar los sentidos otorgados a las prácticas por parte de los entrevistados en un espectro de la temporalidad denominada micro: se trata de la regulación de los tiempos en el momento del consumo. Aquí toman relevancia los conocimientos acerca de los efectos y contraefectos de las sustancias, su duración y el uso que hacen los consumidores de esta información. Cabe aclarar que el foco no está en la calidad de la información con la que cuentan, sino en cómo lo que creen saber o saben es utilizado durante el consumo. En gran medida, lo que "saben" sobre los efectos es "conocido" a partir de la experiencia práctica del consumo. 
Según los distintos relatos de los entrevistados, una vez que deciden consumir, ese momento concreto puede durar entre tres y cinco horas. En ese espacio-tiempo se introduce una regulación que entrecruza el tipo y la duración del efecto de la sustancia, así como un conjunto de evaluaciones prerreflexivas -en el sentido de no conscientes, sin llegar a ser inconscientesy reflexivas ${ }^{(11),(30)}$ acerca del contexto en el que se produce el consumo. Dicho contexto, incluso puede ir modificándose con el pasar del tiempo, lo cual motiva cambios en la elección sobre el consumo de una u otra sustancia. Se repite un consumo para mantener el efecto o para contrarrestarlo. Aquí, saber apreciar el tempo del momento y el contexto relacional son elementos claves para disfrutar del consumo.

Dentro del "momento" del consumo, según los relatos de los entrevistados, se pueden diferenciar diversas fases: una primera, en la cual hay una "entrada en calor" (E29: Mujer, 25 años), que suele realizarse en lo que se denomina "la previa"(50),(51); luego comienza una etapa en la que los efectos de las sustancias están en el punto más álgido, sea para estar "arriba" -en el sentido de activos/estimulados o eufóricos, con capacidad de movimiento para bailar e interactuar-, en "pleno viaje" alucinógeno (E20: Varón, 27 años) o más "tranquis" (E4: Mujer, 24 años), si se trata de reuniones con amigos para cenar o encuentros en espacios al aire libre en casas de fin de semana, plazas o playas.

Entre los entrevistados, además de aprender a administrar las cantidades para extender los efectos durante el tiempo deseado, se presenta la necesidad de las experiencias:

Generalmente, pastillas, tomás media, no tomás una entera porque es un montón. Tomás media y la compartís con otra persona. Así estás como en la misma onda, ¿entendés? Porque si yo tomo una roja y vos tomas una azul, por ahí estamos en cualquiera. Vos estás refeliz y yo no puedo parar de moverme. Por eso, para compartir el sentimiento de estado, que para mí es una de las cosas buenas que tienen las drogas grupalmente... (E2: Varón, 26 años)

Signados por la flexibilidad temporal en su vida cotidiana, "encontrarse" en el "paréntesis temporal" de consumo es muy valorado por estos consumidores, por lo que se despliegan prácticas conscientes orientadas a satisfacer esa sincronización ${ }^{(52)}$.

El momento de consumo suele cortarse con otras sustancias que "bajan" (como el alcohol cuando se consume cocaína) o disminuyen efectos (bebida energizante para "levantar" cuando el nivel de alcohol alcanza una fase depresora). Transitar con mayor o menor "éxito" por estas fases estará condicionado por la preparación previa o por haber accedido a sustancias cuyo efecto es conocido. En la perspectiva de los entrevistados, el acento de los relatos está puesto en los modos por los cuales se concentran en el despliegue de una serie de prácticas que se pueden definir como de "automonitoreo" y que parecen querer demostrar un sentido no problemático de sus prácticas: ser flexibles y dispuestos al cambio; controlar "no ir a destiempo" en un tiempo sin parámetros; conocer el sentido de la ubicación, sabiendo reconocer el momento, la oportunidad; en suma, desplegar un habitus flexible que les permita consumir sin ser consumista: drogarse sin ser "adicto".

\section{A MODO DE CIERRE}

A partir del análisis, en los relatos se pudieron identificar cuatro temporalidades sobre las que los entrevistados despliegan un trabajo reflexivo y en las que están involucrados los usos de sustancias psicoactivas:

- Temporalidades largas: refieren a los sentidos asignados a las prácticas de consumo según la etapa vital o del ciclo de vida en el que se utilizan.

- Temporalidades concretas: moldeadas por la lógica de la sociedad de consumo, definen un ordenamiento del ocio que lo vuelve 
intensivo o intersticial $y$, en esos tiempos inespecíficos, es cuando emergen los consumos de drogas y se evidencian para los consumidores las tensiones cotidianas para definir sus prácticas como "problemáticas" y/o asociadas al "disfrute".

- Ciclos de consumo: cambian según la intensidad del consumo o la elección de la sustancia utilizada, que se ven afectados por la oferta del mercado de drogas, por la propia lógica del consumo desplegada por los entrevistados basada en una búsqueda de cambio constante y, especialmente, por ciclos definidos a partir de una reflexión focalizada en la proximidad/distancia entre un consumo y otro. Esa reflexión les permite controlar el "ritmo" y la "frecuencia" para "no quedar enganchados".

- Temporalidades micro: refieren a regulaciones que se producen una vez que se ha elegido el momento de consumo, y que generan una reflexión sobre cómo utilizar las sustancias, por los efectos de la combinación de unas con otras que posibilitan mayor/menor resistencia, la permanencia en los espacios de ocio y la sincronización de temporalidades individuales con las colectivas.

Por un lado, esto permite señalar cómo las definiciones "expertas" se basan en criterios temporales arbitrarios -en el sentido de no "ser naturales" y "objetivas" sino socialmente definidas- mediante los cuales clasifican a los sujetos como enfermos o sanos que, en nuestra sociedad, y en lo relativo al uso de drogas, se traduce en la pérdida o no de derechos y libertades. Mientras que, por otro lado, en las experiencias de los consumidores, el esfuerzo parece estar focalizado en cumplir con los patrones temporales -no ir a destiempo-, establecer criterios temporales que permitan un automonitoreo tendiente a no fijarse a una sustancia o prácticas repetitivas compulsivas, lo que supone desplegar acciones de forma flexible, ser capaces de ajustarse al momento, o desplegar el consumo según se presente la oportunidad.

Desde una mirada más amplia, los cambios sociales de la modernidad reflexiva, expresados centralmente en los procesos de redefinición y aceleración temporal, no solo obligan a rever categorías que han sido naturalizadas, sino a reconocer su efecto de poder sobre los cuerpos y los sujetos, objeto de intervención desde el campo de la salud. En esta búsqueda de la desnaturalización, la sociología, al ser una ciencia que incomoda, plantea interrogantes a lo obvio e invita a una reflexión que, desde la perspectiva de salud respetuosa de los derechos individuales, sociales, económicos y políticos, es ineludible.

\section{NOTAS FINALES}

a. El Área Metropolitana de Buenos Aires, si bien no se trata de una unidad territorial con entidad política, es una región urbana con una fuerte integración económico-social que comprende la Ciudad Autónoma de Buenos Aires y los 24 partidos del conurbano de la provincia de Buenos Aires.

b. Con el juego de palabras entre doble jornada y doppia presenza (doble presencia), en los años setenta, Laura Balbo caracterizaba la situación de gran parte de las mujeres que se veían obligadas a afrontar la vida laboral y familiar. La idea de presencia, más que de jornada, busca destacar que estas mujeres no solo dedican su tiempo a la actividad laboral, sino que garantizan las necesidades y satisfacen demandas de otros miembros del hogar. $\mathrm{Si}$ bien puede haber diferencias entre un caso y otro, la pauta común es la falta de tiempo para sí. c. Este trabajo presenta una reformulación de uno de los capítulos de mi tesis de doctorado titulada Habitus flexible y modos de subjetivación temporal: análisis sobre los sentidos y las prácticas de los poli-consumidores de drogas del AMBA, defendida en abril de 2015, que no ha sido publicada aún.

d. Se utiliza la Encuesta Nacional sobre Prevalencias de Consumo de Sustancias Psicoactivas (ENPreCoSP) del año 2011, por ser la que cuenta con datos más recientes. Fue diseñada por el Ministerio de Salud de la Nación y el Instituto Nacional de Estadística y Censos (INDEC), relevada a través del Sistema Estadístico Nacional (SEN) coordinado por este último organismo.

e. Para la inclusión en la muestra se consideró a personas que: residan en el AMBA; hayan completado el secundario y estén cursando o tengan pensado concretar una formación universitaria; al menos uno de los progenitores posea nivel educativo secundario completo 
o superior y/o se desempeñen en ocupaciones con personal a cargo; posean un ingreso propio -al menos como complemento de la ayuda familiar o para gastos personales- que garantice una mínima autonomía económica que pueda ser dirigida a las actividades de ocio y al consumo de sustancias.

f. Policonsumo de drogas (polydrug use) engloba diversas modalidades de uso de más de una droga por parte de una misma persona ${ }^{(33)}$. Asimismo, el término "drogas" incluye sustancias que son utilizadas por sus efectos psicoactivos, sean estas legales (como alcohol, tabaco o medicamentos utilizados sin supervisión médica) o ilegales (marihuana, cocaína, etcétera). Los entrevistados incluidos en este trabajo, además de alcohol, tabaco y marihuana, han afirmado consumir una o más de estas sustancias: éxtasis, LSD, cocaína, ketamina, hongos alucinógenos, san pedro, popper, lanza perfumi, psicofármacos sin prescripción médica, viagra y peyote.

g. Este trabajo no se explaya sobre estas tipologías por falta de espacio, por tanto los datos presentados corresponden a los sentidos compartidos por los entrevistados, sin señalar los aspectos de disenso o diferenciación de acuerdo a la tipología utilizada en la investigación completa. En este sentido, los verbatim seleccionados son los que reflejan con mayor claridad los sentidos compartidos por los entrevistados.

h. Esta condición se manifiesta en un patrón con dos elementos: una falla recurrente en los mecanismos de control y una continuación de la/s conducta/s a pesar de sus consecuencias negativas. Entonces, los adictos son personas que tienen dificultad para dejar el hábito de consumir sustancias de forma permanente ${ }^{(53)}$.

i. Nightology es un concepto utilizado en la publicidad de una marca reconocida de whisky que organiza fiestas en un crucero. La noción encierra la idea de conocimiento de las prácticas de la noche. En el contexto de este trabajo, "noche" engloba un conjunto de prácticas de ocio festivo que van más allá de las destinadas al ocio nocturno pero que encierran su "filosofía".

j. Con el concepto de habitus flexible ${ }^{(28)}$ se busca señalar un conjunto de disposiciones que denotan prácticas con un sentido de tempo social cambiante, implica sujetos abiertos a los sucesos, en espera de la realización de lo espontáneo, dispuestos a que se presente la oportunidad. El "sentido del juego" es la flexibilidad, que se vuelve la "razón de ser, pero también una dirección, una orientación, un por-venir" ${ }^{\prime 11)}$.

k. Según la legislación vigente en Argentina, Ley 23.737/89, se puede observar que mientras, por un lado, se penaliza la tenencia para uso personal, quedando a evaluación del juez si la circunstancia permite deducir o no esa situación; por el otro, en el "Fallo Arriola"(54), se invoca el Artículo 19 de la Constitución Nacional, donde se sostiene que "Las acciones privadas de los hombres que de ningún modo ofendan al orden y a la moral pública ni perjudiquen a un tercero, están sólo reservadas a Dios, y exentas de la autoridad de los magistrados. Ningún habitante de la Nación será obligado a hacer lo que no manda la ley, ni privado de lo que ella no prohíbe".

\section{AGRADECIMIENTOS}

Este trabajo ha sido posible gracias a la beca del Consejo Nacional de Investigaciones Científicas y Técnicas (CONICET), de la cual soy beneficiaria desde 2010, bajo la dirección de la Dra. Ana María Medes Diz. Agradezco muy especialmente a Andrés, Fátima, Mora y Candela por su invaluable ayuda.

\section{REFERENCIAS BIBLIOGRÁFICAS}

1. Indij G. Sobre el tiempo. Buenos Aires: La Marca; 2008.

2. Bergmann W. The problem of time in sociology: An overview of the literature on the state of theory and research on the 'Sociology of Time', 1900-82. Time \& Society. 1992;1(1):81-134.

3. Nowotny $\mathrm{H}$. Time and social theory: Towards a social theory of time. Time \& Society. 1992;1(3):421-454.

4. Donabedian A. Una aproximación a la monitorización de la calidad asistencial. Control de Calidad Asistencial. 1991;6(1):1-6.

5. Ong LM, Haes JC, Hoos AM, Lammes FB. Doctorpatient communication: a review of the literature. Social Science \& Medicine. 1995;40(7):903-918.

6. Whittock M, Edwards C, McLaren S, Robinson O. 'The tender trap': Gender, part-time nursing and the effects of 'family-friendly' policies on career advancement. Sociology of Health \& Illness. 2002;24(3):305-326.

7. Domínguez Mon A, Mendes Diz AM, Schwarz P, Camejo M. Usos del tiempo, temporalidades y géneros en contextos. Buenos Aires: Antropofagia; 2012.

8. Zimbardo PG, Boyd JN. Putting time in perspective: A valid, reliable individual-differences metric. Journal of Personality and Social Psychology. 1999;77(6):1271-1288.

9. Rosa H, Scheuerman WE. High-speed society: social acceleration, power, and modernity. Pennsylvania: Pennsylvania State University Press; 2009.

10. Harvey D. La condición de la posmodernidad: Investigación sobre los orígenes del cambio cultural. 2a ed. Buenos Aires: Amorrortu; 2008.

11. Bourdieu P. El sentido práctico. Buenos Aires: Siglo XXI Editores; 2007.

12. Bourdieu P. Argelia 60: Estructuras económicas y estructuras temporales. Buenos Aires: Siglo XXI Editores; 2006.

13. Durkheim E. Las formas elementales de la vida religiosa. México: Colofón; 2000.

14. Sorokin PA, Merton RK. Social time: A methodological and functional analysis. American Journal of Sociology. 1937;42(5): 615-629. 
15. Lewis JD, Weigert AJ. The structures and meaning of social time. Social Forces. 1981;60(2):432-462.

16. Bimbi F. Measurement, quality and social change in reproduction time: The twofold presence of woman and the gift economy. In: Hufton O, Kravaritou Y. Gender and the use of time. Boston: Kluwer Academic Publishers; 1999. p. 151-171.

17. Balbo L. La doppia presenza. Inchiesta. 1978;32:3-7.

18. Coriat B. El taller y el cronómetro: Ensayo sobre el taylorismo, el fordismo y la producción en masa. 11a ed. México DF: Siglo XXI Editores; 1997.

19. Sibilia P. El hombre postorgánico: cuerpo, subjetividad y tecnologías digitales. México DF: Fondo de Cultura Económica; 2005.

20. Segre S. A Weberian theory of time. Time \& Society. 2000;9(2/3): 147-170.

21. Weber M. La ética protestante y el espíritu del capitalismo. 2a ed. México DF: Fondo de Cultura Económica; 2004.

22. Thompson E. Time, work-discipline, and industrial capitalism. Past \& Present. 1967;38(1):56-97.

23. Foucault M. Entre filosofía y literatura: Obras esenciales, volumen I. Barcelona: Paidós; 1999.

24. Foucault M. Vigilar y castigar: Nacimiento de la prisión. Buenos Aires: Siglo XXI Editores; 2002.

25. Foucault M. Estética, ética y hermenéutica: Obras esenciales, volumen III. Barcelona: Paidós; 1999.

26. Binkley S. The work of neoliberal governmentality: Temporality and ethical substance in the tale of two dads. Foucault Studies. 2009;6:60-78.

27. Binkley S. Governmentality, temporality and practice from the individualization of risk to the contradictory movements of the soul. Time \& Society. 2009;18(1):86-105.

28. Sánchez Antelo VIM. Habitus flexible y modos de subjetivación temporal: análisis sobre los sentidos y las prácticas de los poli-consumidores de drogas. [Tesis de doctorado]. Buenos Aires: Universidad de Buenos Aires; 2015.

29. Bourdieu P. ¿Qué significa hablar? 3a ed. Madrid: Akal; 2001.

30. Bourdieu P, Wacquant L. Una invitación a la sociología reflexiva. 2a ed. Buenos Aires: Siglo XXI Editores; 2008.

31. Denzin NK. The Sage handbook of qualitative research. 3a ed. London: SAGE Publications; 2006.

32. Glaser BG, Strauss A. The discovery of grounded theory: Strategies for qualitative research. 3a ed. New Jersey: Transaction Publishers Rutgers; 2006.
33. Ives R, Ghelani P. Polydrug use (the use of drugs in combination): A brief review. Drugs: Education, Prevention, and Policy. 2006;13(3):225-232.

34. CONICET-MECyT. Resolución 2857, CONICET: Lineamientos para el comportamiento ético en las Ciencias Sociales y Humanidades. Buenos Aires: CONICET; 2006

35. Strauss A, Corbin J. Bases de la investigación cualitativa: Técnicas y procedimientos para desarrollar la teoría fundamentada. Antioquia: Editorial Universidad de Antioquia; 1990.

36. Baricco A. Los bárbaros: Ensayo sobre la mutación. Barcelona: Anagrama; 2008.

37. Foucault M. Estrategias de poder: Obras esenciales, volumen II. Barcelona: Paidós; 1999.

38. Bourdieu P. El conocimiento por cuerpos. En: Meditaciones pascalianas. Barcelona: Anagrama; 1999. p. 169-214.

39. Foucault M. Historia de la sexualidad II: El uso de los placeres. 2a ed. Buenos Aires: Siglo XXI Editores; 2010.

40. Moore D. Erasing pleasure from public discourse on illicit drugs: On the creation and reproduction of an absence. International Journal of Drug Policy. 2008;19(5):353-358.

41. O'Malley $P$, Valverde M. Pleasure, freedom and drugs: The uses of 'pleasure' in liberal governance of drug and alcohol consumption. Sociology. 2004;38(1):25-42.

42. Romaní O. Políticas de drogas: prevención, participación y reducción del daño. Salud Colectiva. 2008:4(3):301-318.

43. García López N, Sánchez Antelo VIM, Valente H, Fonseca EP. Nightlife Empowerment and Well-being Implementation Project (NEWIP): primeros resultados del impacto de nuevas políticas de prevención en festivales europeos. Presentado en: XL Jornadas Nacionales Socidrogalcohol. Murcia: Sociedad Científica Española de Estudios sobre el Alcohol, el Alcoholismo y las otras Toxicomanías; 2013.

44. Martínez Oró DP, Pallarés Gómez J. De riesgos y placeres: Manual para entender las drogas. Barcelona: Milenio; 2013.

45. Margulis M. La cultura de la noche. En: La cultura de la noche: la vida nocturna de los jóvenes en Buenos Aires. Buenos Aires: Biblos; 1997. p. 11-30.

46. Sánchez Antelo VIM. Políticas europeas de juventud, ocio y consumo de sustancias psicoactivas. En: Méndez Diz AM, Schwarz PKN, (coord). Juventudes y género: Sentidos y usos del cuerpo, tiempos y espacios en los jóvenes de hoy. Ciudad Autónoma de Buenos Aires: Lugar Editorial; 2012. p. 30-52.

47. Camarotti AC. Música electrónica, escenarios y consumo de éxtasis. Encrucijadas. 2008;(44):1-4. 
48. Camarotti AC. Prácticas, discursos y nuevos espacios de sociabilidad en torno al consumo de éxtasis de jóvenes de sectores medios de la Ciudad de Buenos Aries. [Tesis de doctorado]. Buenos Aires: Universidad de Buenos Aires; 2010.

49. Lenarduzzi V. Placeres en movimiento: Cuerpo, música y baile en la "escena electrónica". Buenos Aires: Aidos Editores; 2012.

50. Teselman C, Laplacette G, Rodríguez Guevara H, Sánchez Antelo V. Consumo de alcohol en adolescentes. En: Experiencias en la prevención y control del consumo de alcohol. Buenos Aires: Ministerio de Salud de la Nación; 2006. p. 79-90.
51. Míguez HA. Alcohol y disponibilidad en la fiesta adolescente. Trastornos Adictivos. 2009;11(1):39-43.

52. Järvinen M, Ravn S. Out of sync: Time management in the lives of young drug users. Time \& Society. 2015; doi:10.1177/0961463X15579577.

53. Beck AT, Wright FD, Newman CF, Liese BS. Terapia cognitiva de las drogodependencias. Buenos Aires: Paidós; 1999.

54. Corte Suprema de Justicia de la Nación. Arriola, Sebastián y otros s/ causa no 9080 . Ciudad Autónoma de Buenos Aires: CSJN; 2009.

\section{FORMA DE CITAR}

Sánchez Antelo V. La dimensión temporal del consumo de drogas: análisis sociológico desde una categoría clave para el estudio de los procesos de salud-enfermedad-atención-cuidado. Salud Colectiva. 2016;12(1):41-54.

Recibido: 9 de julio de 2015 | Versión final: 18 de septiembre de 2015 | Aprobado: 1 de diciembre de 2015

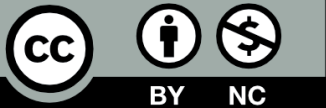

Este obra está bajo una licencia de Creative Commons Reconocimiento-NoComercial 4.0 Internacional. Reconocimiento - Permite copiar, distribuir y comunicar públicamente la obra. A cambio, se debe reconocer y citar al autor original. No Comercial - Esta obra no puede ser utilizada con finalidades comerciales, a menos que se obtenga el permiso.

http://dx.doi.org/10.18294/sc.2016.860 